\title{
Análisis y propuesta de implementación de un observatorio TIC para un conjunto de mipymes de la localidad de Usaquén (Bogotá) en la Universidad de San Buenaventura
}

\author{
Analysis and Proposall for limplementation of a Tics \\ Observatory for a Set of Mipymes of the Usaguên (Bogotál) \\ Locality in the San Buenaventura University
}

\section{Resumen}

Con la incursión de las Tecnologías de la Información y la Comunicación (TIC) en diferentes ámbitos del ser humano, se ha logrado una evolución mayor en cada uno de ellos gracias a las diversas herramientas TIC existentes y en desarrollo que se pueden encontrar. Los procesos de innovación tecnológica permiten a las diferentes organizaciones,

M. Sc. en Telecomunicaciones, Universidad Nacional de Colombia, Ingeniero Electrónico, Escuela Colombiana de Ingeniería, profesor Universidad de San Buenaventura, Bogotá, investigador Grupo Solsytec. E-mail: antorresg@usbbog.edu.co

** Especialista en Negocios y Servicios de Telecomunicaciones, Universidad de San Buenaventura, Bogotá, Ingeniera Electrónica Universidad Pedagógica y Tecnológica de Colombia, profesora, Universidad de San Buenaventura, Bogotá, Investigadora Grupo ICT Research. E-mail: johmartinez@usbbog.edu.co 
sea cual sea su campo de trabajo, generar una dinámica de cambio continua que impacta directamente en las competencias organizativas.

Toda organización que desee incorporar las herramientas de las tecnologías de la información y la comunicación debe generar un plan TIC que considere entre otros aspectos un alineamiento con los objetivos de la empresa, una gestión adecuada de recursos y un monitoreo constante del plan para lo cual debe contarse con personal capacitado para estudiar la naturaleza de la organización, su estructura económica, analizar sus potencialidades y falencias en cuanto a las TIC se refiere y así proponer un plan de incorporación adecuado a la compañía.

El presente documento pretende plantear la creación de un observatorio TIC para un conjunto de micros, pequeñas y medianas empresas (mipymes) de la localidad de Usaquén (Bogotá) en la Universidad de San Buenaventura, basados en el análisis de diferentes observatorios ya creados así como de planes gubernamentales en TIC tanto nacionales como internacionales realizando la propuesta de la conformación del observatorio a partir de su planeación estratégica, metodologías de trabajo y unos planes de capacitación.

\section{Palabras clave}

TIC, observatorio, mipymes, planeación.

\section{Abstract}

The incursion of the information technologies and comunications TIC in different areas of the human being, a major evolution has achieved in each of them thanks to the diverse tools TIC existing and in development that they can find. The processes of technological innovation allow the different organizations, be which is his work camp, to generate a dynamics of change it continues that it affects directly the organizational competitions.

Any organization that wants to incorporate the tools of the TIC must generate a plan TIC that considers between other aspects an alignment with the aims of the company, a suitable management of resources and a constant monitoring of the plan for which one must count with personnel qualified to study the nature of the organization, his economic structure, to analyze his potentials and failings as for the TIC refers and this way to propose a plan of incorporation adapted to the company.

The present document tries to raise the creation of an observatory TIC for a set of small businesses MIPYMES of Usaquén's locality (Bogota) in the San Buenaventura University based on the analysis of different already created observatories as well as of governmental plans in TIC so much national as international realizing the offer of the conformation of the observatory from his strategic planning, methodologies of work and a few plans of training.

\section{Keywords}

TIC, observatories, mipymes, planning. 


\section{Introducción}

El análisis y propuesta de implementación de un observatorio TIC para un conjunto de mipymes de la localidad de Usaquén (Bogotá) presentada en este documento arranca con una descripción general sobre los programas de Ingeniería de Sistemas e Ingeniería de Telecomunicaciones.

La identificación de oportunidades y amenazas surge del análisis de experiencias regionales, nacionales e internacionales acerca de la creación y operación de observatorios tecnológicos, así como del estudio de las políticas, planes y metas del Gobierno nacional (Plan Nacional de TIC, Plan Vive Digital, Plan Nacional de Desarrollo 2010-2014, Política Nacional de Productividad y Competitividad, Sistema Nacional de Ciencia, Tecnología e Innovación, Ley 1286 de 2009) y de organismos internacionales (Unión Internacional de Telecomunicaciones, Foro Económico Mundial, Banco Mundial, entre otros) acerca del uso y apropiación de las TIC.

A continuación, se procede a diseñar el plan estratégico para la creación y funcionamiento del Observatorio. Para ello se inicia con la formulación de la misión y la visión del Observatorio, y se establecen los objetivos estratégicos a partir del análisis de la matriz FODA. También es necesario conceptualizar los campos de trabajo del Observatorio con el fin de determinar los objetivos de las líneas de interés. Con base en eso, es muy importante identificar un conjunto de potenciales asociados para trabajar, en lo posible, de manera colaborativa para fortalecer los resultados así como determinar los recursos necesarios para la creación y puesta en funcionamiento del Observatorio.

Finalmente, se proyectará un programa de capacitación dirigido a un conjunto de mipymes, el cual podrá estar compuesto de cursos de corta duración, organizados a manera de diplomados, cuyo objetivo es motivar el uso de las TIC en los procesos empresariales.

\section{Características generales}

\section{A. Mipymes (micros, pequeñas y medianas empresas)}

Según lo establecido en la Ley 590 de 2000 [1], las mipymes en Colombia, son clasificadas teniendo en cuenta dos características principales: el número de empleados que integran la empresa y la cantidad de activos que genera la empresa, este último, en términos de salarios mínimos mensuales legales vigentes (SMMLV).

\begin{tabular}{|c|c|c|}
\hline TAMAÑo & NÚMERO DE EMPLEADOS & ACTIVOS (SMMLV) \\
\hline Microempresa & $1-10$ & Hasta 500 \\
\hline Pequeña empresa & $11-50$ & Entre 501 y 5.000 \\
\hline Mediana empresa & $51-200$ & Entre 5001 y 30.000 \\
\hline
\end{tabular}

Tabla 1. Clasificación de las mipymes [2] 
En cuanto a las actividades empresariales relacionadas con las mipymes, la Ley 905 de 2004 [3], que modifica la Ley 590 de 2000 define: «Se entiende por micro, pequeña o mediana empresa, toda unidad de explotación económica, realizada por persona natural o jurídica, en actividades empresariales, agropecuarias, industriales, comerciales o de servicio, rural o urbano, incluyendo los artesanos».

\section{B. Observatorio TIC}

Se entiende por Observatorio TIC, un espacio en el cual se trabajan proyectos relacionados con el uso, implementación, aplicación y apropiación de las Tecnologías de la Información y las Comunicaciones (TIC), con el fin de potencializar el desarrollo tecnológico en entidades de diferentes áreas generando impactos económicos y sociales.

\section{Análisis de los programas de Sistemas y Telecomunicaciones de la Universidad de San Buenaventura, Bogotá}

\section{Ingeniería de Sistemas}

Existen diferentes definiciones para explicar la Ingeniería de Sistemas.

En la Unión Europea se denomina Ingeniería Informática, definida por ACM [4], como:

«[...] toda actividad orientada hacia los objetivos, que se beneficia de la creación del computador. Por lo tanto la computación incluye el diseño y construcción de sistemas de hardware y software, para un amplio rango de propósitos:

- Procesar, estructurar y administrar varias clases de información.

- Hacer estudios científicos usando computadores.

- Hacer sistemas de computador que se comporten inteligentemente.

- Crear y usar las comunicaciones y los medios de entretenimiento.

- Encontrar y tomar información relevante para un propósito general».

En Estados Unidos se conoce como Ingeniería de Sistemas, aplicando la definición dada por el Institute of Electrical and ElectronicsEngineers (IEEE), la cual establece que: «Ingeniería de Sistemas es la aplicación de las ciencias matemáticas y físicas para desarrollar sistemas que utilicen económicamente los materiales y fuerzas de la naturaleza para el beneficio de la humanidad» [5].

En Colombia, la Red de Decanos y Directores de Ingeniería de Sistemas (REDIS) establece que la Ingeniería de Sistemas: «busca a través de la combinación de varias disciplinas, crear y estudiar sistemas complejos de información tecnológica que se materializan en programas de computación no tangibles conocidos como software» [6].

De acuerdo con las definiciones dadas anteriormente, se puede establecer que la Ingeniería de Sistemas, está concebida como un área en la cual se emplean los computadores 
para el manejo de información con el fin de optimizar procesos, mediante la integración y uso adecuado de Hardware y Software.

\section{Ingeniería de Telecomunicaciones}

Según la Unión Internacional de Telecomunicaciones (UIT), se define telecomunicaciones como: «Toda transmisión, emisión o recepción de signos, señales, escritos, imágenes, sonidos o informaciones de cualquier naturaleza por hilo, radioelectricidad, medios ópticos u otros sistemas electromagnéticos» [7].

La Ingeniería de Telecomunicaciones se encuentra definida por el DANE dentro del extracto de la clasificación internacional uniforme de ocupaciones 088 adaptada para Colombia [8] en el grupo 2: profesionales científicos e intelectuales, subgrupo 2143: ingenieros eléctricos, electrónicos, de telecomunicaciones y afines. En este subgrupo se especifica la función del ingeniero de estos campos, así: «Proyectan, diseñan, desarrollan, dirigen y vigilan la construcción, funcionamiento, conservación y reparación de instalaciones de producción, transporte y distribución de energía eléctrica, equipos y aparatos eléctricos y electrónicos o de telecomunicaciones, e investigan y asesoran al respecto» [8].

De acuerdo con las definiciones dadas anteriormente, se puede establecer que la Ingeniería de Telecomunicaciones, es una profesión que se dedica a la transmisión y recepción de información a través de señales electromagnéticas por medios guiados o no guiados.

\section{Matriz Dofa}

La matriz de Debilidades, oportunidades, fortalezas y amenazas (Dofa), es una herramienta que ayuda a realizar el análisis de recursos de una entidad, permitiendo medir las capacidades y limitaciones que pueden generar implicaciones positivas o negativas en el futuro de la organización.

«Los encabezados de la matriz proveen un buen marco de referencia para revisar la estrategia, posición y dirección de una empresa, propuesta de negocios, o idea» [9].

\section{Fortaleza competitiva}

Hace referencia a actividades que la entidad hace bien o a los atributos que hacen que sea competitiva dentro de un mercado.

\section{Debilidad competitiva}

Cuando se habla de debilidades competitivas, se está haciendo referencia a carencias o actividades mal hechas, teniendo como referencia a otras agrupaciones o instituciones que realizan las mismas actividades, y que la ponen en desventaja [10].

\section{Oportunidad}

Se habla de oportunidades, cuando después de hacer un análisis externo o del entorno, se identifican factores positivos, que pueden ser aprovechados, en la mayoría de los casos, 
son actividades que las organizaciones de la competencia, no realizan o tienen debilidades en el desarrollo de las mismas.

\section{Amenaza}

Al igual que las oportunidades, estas se identifican luego de realizar el análisis del entorno o externo, pero en este caso, se hace referencia a situaciones negativas que pueden afectar un proyecto o a la entidad. En estos casos, la organización, diseña estrategias que permitan afrontar los posibles inconvenientes que se presenten.

A partir de estas descripciones se puede llegar a conclusiones que permitan ver el comportamiento general de la organización, y a partir de este, realizar acciones estratégicas que conlleven a nuevas perspectivas y mejoras fundamentales dentro de la institución.

\section{Observatorios TIC a nivel nacional e internacional}

Teniendo en cuenta el creciente desarrollo y el interés en las temáticas referentes a Tecnologías de la Información y las Comunicaciones, es necesario analizar el comportamiento de los componentes de la sociedad, en particular, el que presentan las micro, pequeñas y medianas empresas (mipymes).

A nivel internacional y nacional, se tiene una perspectiva de análisis que marca la tendencia de los observatorios TIC.

\section{A. Análisis de los observatorios TIC}

A partir de los observatorios TIC nacionales e internacionales, es necesario identificar los ejes de acción de cada uno de ellos, las entidades que los rigen o regulan y los proyectos que han trabajado, esto con el fin de poder realizar el análisis del entorno que alimentará la matriz Dofa que permitirá plantear los objetivos estratégicos alimentará los planes de acción que trabajará el Observatorio TIC de la Universidad de San Buenaventura.

\section{Observatorios internacionales}

\begin{tabular}{|l|l|}
\hline \multicolumn{1}{|c|}{ Nombre } & \multicolumn{1}{|c|}{ Observatorio Nacional de las Telecomunicaciones y la Sociedad de la Información (ONTSI) } \\
\hline País & España. \\
\hline \hline Página Web & www.ontsi.red.es/ontsi/ \\
\hline \hline Misión & $\begin{array}{l}\text { Su misión es la elaboración de estudios, el seguimiento y evaluación de políticas públicas para una } \\
\text { colaboración y coordinación con el sector público y privado en cuanto la sociedad de la Información } \\
\text { esta entidad promotora de las tecnologías es líder en España por sus grandes aplicaciones en la } \\
\text { sociedad. }\end{array}$ \\
\hline \hline Temática que trabaja & $\begin{array}{l}\text { Economía digital. } \\
\text { - Sociedad digital. } \\
\text { - Servicios públicos digitales. }\end{array}$ \\
\hline
\end{tabular}

Tabla 2. Características Observatorio Nacional de las Telecomunicaciones y la Sociedad de la Información (ONTSI) [11]. 


\begin{tabular}{|l|l||}
\hline \multicolumn{1}{|c|}{ Nombre } & \multicolumn{1}{c|}{ Cesalvi: Observatorio Valenciano para la Sociedad Tecnológica y del Conocimiento. } \\
\hline País & España (Valencia) \\
\hline \hline Página Web & http://www.cevalsi.org \\
\hline \hline Misión & $\begin{array}{l}\text { Dotar a la comunidad valenciana de un centro de excelencia que acumule y gestione los } \\
\text { conocimientos y las experiencias relacionados con la Sociedad de la Información. }\end{array}$ \\
\hline \hline \multirow{2}{*}{ Temática que trabaja } & - Sociales \\
& - Económicos \\
\hline
\end{tabular}

Tabla 3. Características Cesalvi: el Observatorio Valenciano para la sociedad Tecnológica y del Conocimiento [12].

\begin{tabular}{|l|l||}
\hline \multicolumn{1}{|c|}{ Nombre } & \multicolumn{1}{c|}{ Observatorio TIC Aragonés de la Sociedad de la Información } \\
\hline País & España (Aragonés) \\
\hline \hline Página Web & www.observatorioaragones.org/ \\
\hline Misión & $\begin{array}{l}\text { Generar estudios estadísticos de almacenamiento de información y análisis y lograr presentar } \\
\text { resultados sistemáticos de la implementación de las tecnologías a la sociedad de información. }\end{array}$ \\
\hline \hline Temática que trabaja & - Sociales \\
& - Económicos \\
\hline
\end{tabular}

Tabla 4. Características Observatorio TIC Aragonés de la Sociedad de la Información [13].

\begin{tabular}{|l|l||}
\hline Nombre & Observatorio INTECO de Seguridad de la Información \\
\hline País & España. \\
\hline \hline Página Web & http://wwwinteco.es \\
\hline \hline Misión & $\begin{array}{l}\text { Asesoramiento, promoción y difusión de proyectos tecnológicos para la sociedad de la } \\
\text { información, difundiendo la cultura de la seguridad y la confianza informática en los ciudadanos, } \\
\text { empresas y administraciones españolas }\end{array}$ \\
\hline \hline Temática que trabaja & $\begin{array}{l}\text { Presenta informes anuales realizados por el Instituto Nacional de Tecnologías de la Comunicación, } \\
\text { disponiendo de un plan de actividades con el objetivo de crear conocimiento especializado en la } \\
\text { sociedad acerca de la seguridad informática, como también la elaboración de recomendaciones y } \\
\text { propuestas que sean acordes a tendencia válida para la toma de decisiones en el sector público. }\end{array}$ \\
\hline
\end{tabular}

Tabla 5. Características Observatorio INTECO de Seguridad de la Información [14].

\begin{tabular}{|l|l||}
\hline Nombre & Observatorio Ministerio de Ciencia, Tecnología e Innovación \\
\hline País & Argentina \\
\hline \hline Página Web & http://www.mincyt.gov.ar \\
\hline Misión & $\begin{array}{l}\text { Aumentar la ciencia tecnológica con el fin de generar un modelo productivo para la sociedad y } \\
\text { la industria, promoviendo el crecimiento de la tecnología e innovación a través del aumento de } \\
\text { salarios de investigación e infraestructura. }\end{array}$ \\
\hline \hline Temática que trabaja & $\begin{array}{l}\text { El ministerio presenta anualmente resúmenes ejecutivos que recopilan los datos más relevantes sobre el } \\
\text { trabajo por el Ministerio de Ciencia, Tecnología e Innovación Productiva y sus organismos dependientes. }\end{array}$ \\
\hline
\end{tabular}

Tabla 6. Características Observatorio Ministerio de Ciencia, Tecnología e Innovación [15].

\begin{tabular}{|l|l|}
\hline Nombre & Observatorio de la Sociedad de la Información de Navarra \\
\hline País & España (Navarra) \\
\hline \hline Página Web & www.cfnavarra.es/observatoriosi/praina.htm \\
\hline \hline Misión & $\begin{array}{l}\text { Este programa tiene como iniciativa la colaboración a la Comisión Europea y entidades privadas y } \\
\text { públicas en cuanto el desarrollo de proyectos innovadores. }\end{array}$ \\
\hline \hline Temática que trabaja & $\begin{array}{l}\text { El Observatorio para la Sociedad de la Información en Navarra es un instrumento que permite } \\
\text { obtener y analizar información sobre el grado de desarrollo y utilización de las Tecnologías de la } \\
\text { Información y la Comunicación en la Sociedad Navarra, de forma sistemática. }\end{array}$ \\
\hline
\end{tabular}

Tabla 7. Características Observatorio de la Sociedad de la Información de Navarra [16]. 


\begin{tabular}{|l|l|}
\hline Nombre & Observatorio Unesco \\
\hline & Organización Mundial \\
\hline \hline Página Web & http://www.unesco.org/new/es/unesco/about-us/who-we-are/introducing-unesco/ \\
\hline \hline Misión & $\begin{array}{l}\text { La misión de la Unesco es aportar a la consolidación de la paz, la eliminación de la pobreza, el } \\
\text { desarrollo sostenible y el diálogo intercultural mediante la educación, las ciencias, la cultura, la } \\
\text { comunicación y la información. }\end{array}$ \\
\hline \hline \multirow{2}{*}{ Temática que trabaja } & $\begin{array}{l}\text { - Igualdad entre hombres y mujeres } \\
\text { - Una la educación de calidad para todos }\end{array}$ \\
& $\begin{array}{l}\text { - Conocimiento científico al desarrollo sostenible } \\
\text { - Los nuevos problemas éticos y sociales }\end{array}$ \\
\hline
\end{tabular}

Tabla 8. Características Observatorio Unesco [17].

\begin{tabular}{|l|l|}
\hline Nombre & Observatorio de las Tecnologías de la Información y de la Comunicación (OTIC) \\
\hline País & Chile \\
\hline \hline Página Web & www.observatoriotic.gob.cl \\
\hline \hline Misión & $\begin{array}{l}\text { En busca de apropiarse del uso de las Tecnologías de la información y de la comunicación para uso } \\
\text { y aplicación en ámbitos sociales, educativos e industriales dando cavidad a un Chile desarrollado en } \\
\text { tecnología y conocimiento. }\end{array}$ \\
\hline \hline Temática que trabaja & $\begin{array}{l}\text { Información y estrategias de planeación de acciones digitales realizadas por entidades públicas, } \\
\text { privadas y académicas. }\end{array}$ \\
\hline
\end{tabular}

Tabla 9. Características Observatorio de las Tecnologías de la Información y de la Comunicación (OTIC) [18].

\begin{tabular}{|l|l|}
\hline Nombre & $\begin{array}{l}\text { Observatorio de la Sociedad de la Información y el Conocimiento de la República Dominicana } \\
\text { (OSIC-RD) }\end{array}$ \\
\hline País & República Dominicana \\
\hline \hline Página Web & http://osicrd.one.gob.do \\
\hline \hline Misión & $\begin{array}{l}\text { Su misión principal es convertirse en un centro de referencia para la medición, análisis, evaluación } \\
\text { y difusión de información sobre el desarrollo de la Sociedad de la Información y el Conocimiento en } \\
\text { República Dominicana. }\end{array}$ \\
\hline \hline Temática que trabaja & $\begin{array}{l}\text { Este organismo es creado en abril del 2005; el observatorio funciona como carácter informativo y } \\
\text { de conocimiento tecnológico para la región de la República Dominicana }\end{array}$ \\
\hline \hline
\end{tabular}

Tabla 10. Características Observatorio de la Sociedad de la Información y el Conocimiento de la República Dominicana [19].

\begin{tabular}{|l|l|}
\hline Nombre & Observatorio Andino de las Tecnologías de la Información y la Comunicación (OBATIC). \\
\hline País & Comunidad Andina (Colombia, Perú, Ecuador y Venezuela). \\
\hline \hline Página Web & $\begin{array}{l}\text { www.intranet.comunidadandina.org/.../sg_re_ } \\
\text { obatic _ i informe.doc }\end{array}$ \\
\hline \hline Misión & $\begin{array}{l}\text { Posicionar las Tecnologías de Información y de Comunicación es una colaboración mutua entre los } \\
\text { países que la conforman, transparencia y fidelidad de la información. }\end{array}$ \\
\hline \hline Temática que trabaja & $\begin{array}{l}\text { Trabaja por medio de recurso de información de la cual recopila procesa y difunde planes proyectos } \\
\text { y servicios privados o públicos en tecnologías de la información y de la comunicación de los países } \\
\text { que hacen parte de la comunidad andina. }\end{array}$ \\
\hline
\end{tabular}

Tabla 11. Características Observatorio Andino de las Tecnologías de la Información y la Comunicación [20] 


\section{Observatorios nacionales}

\begin{tabular}{|l|l||}
\hline Nombre & Observatorio Colombia Digital \\
\hline País & Colombia \\
\hline \hline Página Web & https://www.colombiadigital.net \\
\hline \hline \multirow{3}{*}{ Misión } & $\begin{array}{l}\text { Proveniente de consolidar el uso y apropiación de las Tecnologías de la Información y de la } \\
\text { Comunicación, con fines específicos de implementación, uso, aplicación y apropiación de las TIC } \\
\text { generando un trato con la tecnología y potencializándola para el desarrollo del país, en diversos } \\
\text { ámbitos y contextos ofreciéndole un efecto positivo a varios niveles }\end{array}$ \\
\hline \hline \multirow{3}{*}{ Temática que trabaja } & $\begin{array}{l}\text { Económico } \\
\text { Social } \\
\text { Educativo } \\
\text { El Estado }\end{array}$ \\
\hline
\end{tabular}

Tabla 12. Características Observatorio Colombia Digital [21].

\begin{tabular}{|l|l||}
\hline Nombre & Observatorio de la Universidad Nacional de Colombia, sede Bogotá \\
\hline País & Colombia. \\
\hline \hline Página Web & $\begin{array}{l}\text { http://wwwing.unal.edu.co/GRIEGO/ Actualmente no cuenta con página web, pero el Grupo de } \\
\text { Investigación en Gestión y Organizaciones (GRIEGO) es el encargado del desarrollo de creación y } \\
\text { desarrollo del Observatorio }\end{array}$ \\
\hline \hline Temática que trabaja & $\begin{array}{l}\text { Apropiación tecnológica } \\
\text { Sistemas de información }\end{array}$ \\
\hline
\end{tabular}

Tabla 13. Características Observatorio de la Universidad Nacional de Colombia, sede Bogotá [22].

\begin{tabular}{|l|l||}
\hline Nombre & Observatorio de Educación del Caribe Colombiano \\
\hline País & Colombia \\
\hline \hline Página Web & http://www.uninorte.edu.co/web/observaeduca/ \\
\hline \hline Misión & $\begin{array}{l}\text { Proporcionar, dinamizar y socializar informaciones y conocimientos en educación, que } \\
\text { contribuyan a la mejora de los procesos educativos y desarrollo social de los habitantes del } \\
\text { Caribe colombiano. }\end{array}$ \\
\hline \hline Temática que trabaja & $\begin{array}{l}\text { Documentos informativos de actualidad, investigaciones y proyectos e innovación que se } \\
\text { ha generado por medio de esta iniciativa que busca posicionar la educación en el Caribe } \\
\text { colombiano. }\end{array}$ \\
\hline
\end{tabular}

Tabla 14. Características Observatorio de Educación del Caribe Colombiano [23].

\begin{tabular}{|l|l||}
\hline Nombre & Observatorio Medellín Digital \\
\hline País & Colombia. \\
\hline \hline Página Web & http://www.medellindigital.gov.co/ \\
\hline \hline Misión & $\begin{array}{l}\text { Ubicar a Medellín como ciudad digital interactuando en el sector del desarrollo social, abrirle las } \\
\text { puertas a sectores como la educación, el emprendimiento, el gobierno en línea y la cultura. }\end{array}$ \\
\hline \hline Temática que trabaja & $\begin{array}{l}\text { Se trata de una herramienta «viva», con actualizaciones constantes y que reflejará en cada } \\
\text { momento los avances que la ciudad de Medellín realiza en el campo de las TIC y la Sociedad del } \\
\text { Conocimiento. }\end{array}$ \\
\hline
\end{tabular}

Tabla 15. Características Observatorio Medellín Digital [24]. 


\begin{tabular}{|l|l|}
\hline Nombre & Observatorio de Educación y Pedagogía \\
\hline País & Colombia \\
\hline \hline Página Web & http://www.idep.edu.co/cartografia/opes/index.html \\
\hline \hline Misión & Promoviendo los procesos del sistema educativo y perfeccionando a los docentes y directivos. \\
\hline \hline & - Educación \\
Temática que trabaja & : Población vulnerable \\
& - Hogares \\
& - Ciudad \\
\hline
\end{tabular}

Tabla 16. Características Observatorio de Educación y Pedagogía [25].

\begin{tabular}{|l|l|}
\hline Nombre & Observatorio de Sociedad, Gobierno y Tecnologías de la Información (TIC ). \\
\hline País & Colombia. \\
\hline \hline Página Web & http://observatics.edu.co/ \\
\hline \hline Misión & $\begin{array}{l}\text { Ser el primer centro de investigación sobre la sociedad de la información, sociedad del } \\
\text { conocimiento y ciudades inteligentes en Colombia y América Latina en el año } 2015 .\end{array}$ \\
\hline \hline Temática que trabaja & $\begin{array}{l}\text { Gestión de la información y redes sociales; Gestión documental en el entorno digital; Territorios y } \\
\text { ciudades inteligentes, entre otros. } \\
\text { Consultoría con el fin de asesorar a los entes territoriales y a las entidades de los sectores público y } \\
\text { privado en la implementación de proyectos de tecnologías de información y comunicación (tic). }\end{array}$ \\
\hline
\end{tabular}

Tabla 17. Características Observatorio de Sociedad, Gobierno y Tecnologías de la Información (TIC) [26].

\begin{tabular}{|l|l|}
\hline Nombre & Observatorio Colombiano de Ciencia y Tecnología (OCyT) \\
\hline País & Colombia. \\
\hline \hline Página Web & http://ocyt.org.co/html/ \\
\hline \hline \multirow{3}{*}{ Misión } & $\begin{array}{l}\text { Producir conocimiento sobre la dinámica y el posicionamiento del sistema mediante el diseño, } \\
\text { producción, integración, interpretación y difusión de estadísticas e indicadores, para orientar y } \\
\text { evaluar las políticas y la acción de los diversos actores del SNCTI. }\end{array}$ \\
\hline \hline \multirow{3}{*}{ Temática que trabaja } & $\begin{array}{l}\text { B Bibliometría } \\
\text { - Inversión }\end{array}$ \\
& $\begin{array}{l}\text { - Recursos humanos } \\
\text { - Apropiación }\end{array}$ \\
\hline
\end{tabular}

Tabla 18. Características Observatorio Colombiano de Ciencia y Tecnología (OCyT) [27].

\section{B. Planes, metas e indicadores para la apropiación de las tic}

Después de identificar las características de los observatorios, es necesario identificar los planes, metas y proyectos que a nivel internacional y nacional, fomentan la apropiación de TIC en la sociedad

\section{Planes internacionales}

\subsection{Foro Económico Mundial}

«El WorldEconomicForum es una organización internacional independiente comprometida con el mejoramiento de la situación mundial, que genera asociaciones entre líderes 
empresariales, políticos y académicos, entre otros líderes de la sociedad, para definir la agenda global y las agendas regionales e industriales» [28]. Es una entidad sin ánimo de lucro cuya sede principal se encuentra en Ginebra, Suiza. Como principal misión en cuanto al área tecnológica se refiere, el Foro Económico Mundial pretende fomentar la expansión en las TIC manteniendo el enfoque en el desarrollo económico sin afectar a la sociedad.

El interés principal del Foro Económico Mundial dentro de su participación empresarial se enfoca en tres sectores de interés que son:

- Tecnologías de la Información y Nuevos Medios

- Energía y Medio Ambiente

- Ciencias de la Vida y la Salud

Las empresas deben causar un impacto satisfactorio para la sociedad en cuanto el desarrollo de las tecnologías impulsando el cambio en cuanto a fortalecimiento de las telecomunicaciones, nuevos medios de comunicación, energía y salud y avances científicos tecnológicos.

El personal encargado es evaluado y/o renovado anualmente por medio de un proceso selectivo formado por expertos en tecnologías, así como empresarios del Foro que evalúan los candidatos según el programa. El Foro tiene actualizada toda su información tecnológica detallada de los estudios que se han realizado.

\subsection{El Banco Mundial}

El Banco Mundial fue fundado en 1944. Es una entidad integrada por cinco instituciones de desarrollo, «su misión evolucionó desde el Banco Internacional de Reconstrucción y Fomento (BIRF) como facilitador de la reconstrucción y el desarrollo de posguerra al mandato actual de aliviar la pobreza en el mundo, coordinándose muy de cerca con su afiliado, la Asociación Internacional de Fomento» [29].

El Banco Mundial cuenta con varios profesionales en diferentes áreas, entre ellos, economistas, ingenieros analistas financieros, expertos en políticas públicas y científicos sociales entre otros, estos están distribuidos en diferentes oficinas de distintos países. El objetivo que resalta por parte del Banco en su trabajo es luchar contra la pobreza a través de un proceso de globalización inclusivo y sostenible.

Las cinco instituciones que hacen parte de esta organización son:

1. El Banco Internacional de Reconstrucción y Fomento (BIRF): concede préstamos a gobiernos de países de bajo y mediano ingreso.

2. La Asociación Internacional de Fomento (AIF): concede préstamos a gobiernos sin interés, hace donaciones a países pobres. 
3. La Corporación Financiera Internacional (IFC): es la mayor institución internacional de desarrollo dedicada exclusivamente al sector privado, ayudando a países en desarrollo.

4. Organismo Multilateral de Garantía de Inversiones (MIGA), su principal tarea es promover la inversión extranjera directa en los países en desarrollo.

5. El Centro Internacional de Arreglo Diferencias Relativas a Inversiones (CIADI), ayuda a conciliar disputas sobre inversionistas.

El desarrollo tecnológico de los países les abre las puertas al mundo para poder acceder a las Tecnologías de la Información y de la Comunicación a través de distintos dispositivos brindándole mejores oportunidades a la ciudadanía y a medios sociales. El Banco Mundial es un mecanismo de desarrollo a la apropiación tecnológica promoviendo el aprendizaje estructurado e intercambio de conocimientos.

Algunos de los proyectos que el Banco Mundial ha desarrollado en cuanto a las Tecnologías de la Información y la Comunicación, son:

- Programa Red de Seguridad de Base Tecnológica, transforma vidas de los más pobres en Pakistán: el Banco Mundial ha iniciado el proyecto de pagos a través de tarjetas de débito para las familias elegibles. Las tarjetas pueden ser utilizadas en cualquier cajero automático (ATM) o en la red del banco asociado a cobrar el pago de beneficios.

- La revolución móvil como se ve en el este de Asia: el proyecto promueve el uso de tecnologías móviles para hacer uso de bancos virtuales, realizar transacciones, consultas, balances monetarios y demás transacciones bancarias.

\subsection{Unión Internacional de Telecomunicaciones (UIT)}

La Unión Internacional de Telecomunicaciones, una entidad de las Naciones Unidas diseñada para fortalecer las Tecnologías de la Información y la Comunicación mediante la correcta y eficiente asignación del espectro electromagnético y orbitas satelitales, garantizando a los usuarios una interconexión global con calidad. La UIT trabaja por mejorar el acceso de las TIC A las comunidades marginadas del mundo y se compromete a interconectar todo el mundo. Dentro de sus políticas se incluyen regulaciones en TIC.

De acuerdo con la UIT: «Hoy en día, todo lo que hacemos se sustenta en las TIC. Nos ayudan a organizar y a controlar los servicios de emergencia, el abastecimiento de agua, las redes eléctricas y las cadenas de distribución de alimentos. Se utilizan como soporte para la atención médica, la enseñanza, los servicios públicos, los mercados financieros, las redes de transporte y la gestión medioambiental. Y permiten a la gente comunicarse en todo momento y casi desde cualquier lugar con sus colegas, amigos y familiares» [30].

La UIT ha creado planes de fortalecimiento y ejecución de TIC para implementación nacional, regional y mundial, todo esto gestionado por aspectos administrativos y finan- 
cieros, incluyendo prestación de servicios de conferencia planificación, y organización en servicios de TIC.

La misión de la UIT es proporcionar servicios de alta calidad y eficiente, consolidándola en la prestación de asistencia técnica, creación de proyecto y desarrollo de equipos de telecomunicaciones, para lo cual se desenvuelve en tres áreas principales:

1. El Sector de Radiocomunicaciones de la UIT, (UIT R) coordina todo el conjunto de servicios de radiocomunicaciones y se encarga de la gestión internacional del espectro de frecuencias radioeléctricas y las órbitas de los satélites.

2. Las normas de la UIT (conocidas como Recomendaciones) son fundamentales para el funcionamiento de las actuales redes y servicios de TIC como lo son el acceso a Internet, los protocolos de transporte, la compresión de voz y vídeo, las redes domésticas, etc.

3. El Sector de Desarrollo de las Telecomunicaciones de la HIT (HIT D) tiene como objetivo apoyar a los entes interesados en ingresar o aumentar la presencia en mercados emergentes mediante las TIC. La HIT, que tiene como una de sus finalidades «reducir la brecha digital», impulsa varias iniciativas importantes como los eventos «Tú Connect» o «Conectar una escuela, conectar una comunidad».

\section{Planes nacionales}

\begin{tabular}{|c|c|}
\hline Nombre del Plan o Proyecto & Plan Nacional de las Tecnologías de la Información y de la Comunicación \\
\hline Vigencia & $2008-2019$ \\
\hline Entidad responsable & Ministerio de Comunicaciones de la República de Colombia. \\
\hline Ejes de acción & $\begin{array}{ll}\text { - } & \text { Comunidad } \\
\text { - } & \text { Invesco regulagación } \\
\text { - } & \text { Gobierno en línea } \\
\text { - } & \text { Educación } \\
\text { - } & \text { Salud } \\
\text { - } & \text { Justicia } \\
\end{array}$ \\
\hline Metas & $\begin{array}{l}\text { Busca que al final de este período, todos los colombianos se informen y se comuniquen } \\
\text { haciendo uso eficiente y productivo de las TIC, para mejorar la inclusión social y aumentar la } \\
\text { competitividad. }\end{array}$ \\
\hline Indicadores & Según los ejes trabajados \\
\hline Estrategias & De acuerdo con cada eje \\
\hline
\end{tabular}

Tabla 19. Características Plan Nacional de las Tecnologías de la Información y de la Comunicación [31].

\begin{tabular}{|l|l|}
\hline Nombre del Plan o Proyecto & Vive Digital Colombia \\
\hline Vigencia & $2011-2014$ \\
\hline \hline Entidad responsable & $\begin{array}{l}\text { Ministerio de las Tecnologías de la Información y la Comunicación de la República de } \\
\text { Colombia. }\end{array}$ \\
\hline \hline \multirow{3}{*}{ Ejes de acción } & $\bullet \quad$ Infraestructura \\
& $\begin{array}{l}\text { - Servicios } \\
\text { Aplicaciones }\end{array}$ \\
\hline \hline Metas & $\begin{array}{l}\text { Impulsarios la masificación del uso de Internet, para dar un salto hacia la prosperidad } \\
\text { democrática. }\end{array}$ \\
\hline
\end{tabular}




\begin{tabular}{|l|l|}
\hline Nombre del Plan o Proyecto & Vive Digital Colombia \\
\hline Indicadores & De acuerdo con cada eje de acción \\
\hline \hline Estrategias & Aplicación del modelo Ecosistema Digital \\
\hline
\end{tabular}

Tabla 20. Características Plan Vive Digital Colombia [32].

\begin{tabular}{|c|c|}
\hline Nombre del Plan o Proyecto & $\begin{array}{l}\text { Documento Conpes } 3670 \text { «Lineamientos de política para la continuidad de los programas } \\
\text { de acceso y servicio universal a las tecnologías de la información y las comunicaciones». }\end{array}$ \\
\hline Vigencia & En vigencia desde 2010 \\
\hline Entidad responsable & $\begin{array}{l}\text { Consejo Nacional de Política Económica y Social (Conpes) - Departamento Nacional de } \\
\text { Planeación. }\end{array}$ \\
\hline Ejes de acción & $\begin{array}{l}\text { Distribución de responsabilidades para la financiación del uso y acceso de las tic } \\
\text { 1. Continuidad de provisión de la conectividad en los puntos ya instalados del Programa } \\
\text { Compartel. } \\
\text { 2. Expansión del Servicio de Conectividad - Nuevas conexiones } \\
\text { 3. Provisión de soluciones integrales de tic para la educación básica, media y media } \\
\text { técnica. } \\
\text { - Desarrollo de acciones complementarias para atender cambios y rezagos tecnológicos y } \\
\text { la protección del medio ambiente. }\end{array}$ \\
\hline Metas & $\begin{array}{l}\text { Definir los lineamientos de política para la continuidad de las iniciativas que promueven el } \\
\text { acceso, uso y aprovechamiento de las TIC, de manera coordinada entre los programas del } \\
\text { Ministerio de Tecnologías de la Información y las Comunicaciones y demás instancias del } \\
\text { Gobierno. }\end{array}$ \\
\hline Indicadores & Según ejes de acción \\
\hline Estrategias & $\begin{array}{l}\text { Generación e implementación de programas que mejoren el uso y apropiación de las TIC en la } \\
\text { sociedad. }\end{array}$ \\
\hline
\end{tabular}

Tabla 21. Características Documento Conpes 3670 «Lineamientos de política para la continuidad de los programas de acceso y servicio universal a las tecnologías de la información y las comunicaciones» [33].

\begin{tabular}{|c|c|}
\hline Nombre del Plan o Proyecto & $\begin{array}{l}\text { Ley } 1286 \text { de } 2009 \text { «Por la cual se modifica la Ley } 29 \text { de 1990, se transforma a Colciencias } \\
\text { en Departamento Administrativo, se fortalece el Sistema Nacional de Ciencia, Tecnología e } \\
\text { Innovación en Colombia y se dictan otras disposiciones». }\end{array}$ \\
\hline Vigencia & En vigencia desde 2009 \\
\hline Entidad responsable & Sistema Nacional de Ciencia, Tecnología e Innovación. \\
\hline Ejes de acción & $\begin{array}{l}\text { - } \quad \text { Aumentar la demanda científica. } \\
\text { - } \quad \text { Incorporar la investigación científica } \\
\text { - Integrar los sectores tecnológicos como estrategia para el desarrollo del país. } \\
\text { - } \quad \text { Fortificar el ámbito tecnológico e innovación internacional. } \\
\text { - } \quad \text { Gortalecer la calidad en educación. }\end{array}$ \\
\hline Metas & $\begin{array}{l}\text { Fortalecer el Sistema Nacional de Ciencia y Tecnología para lograr un modelo productivo en } \\
\text { la ciencia, la tecnología y la innovación, desarrollo agregado a los productos y servicios de } \\
\text { nuestra economía y propiciar el desarrollo productivo y una nueva industria nacional. }\end{array}$ \\
\hline Estrategias & $\begin{array}{l}\text { - } \quad \text { Explorar e investigar escenarios internacionales. } \\
\text { - } \quad \text { Mejorar la productividad y la competitividad nacional. } \\
\text { - } \quad \text { Investigar e innovorimiento científico. } \\
\text { - Integrar la cultura tecnológica e innovadogía. } \\
\text { - } \quad \text { Desarrollo del conocimiento de la ciencia en instituciones educativas de la sociedad } \\
\text { - } \quad \text { colombiana. }\end{array}$ \\
\hline
\end{tabular}

Tabla 22. Características Ley 1286 de 2009 «Por la cual se modifica la Ley 29 de 1990, se transforma a Colciencias en Departamento Administrativo, se fortalece el Sistema Nacional de Ciencia, Tecnología e Innovación en Colombia y se dictan otras disposiciones» [34]. 


\section{Planeación estratégica del Observatorio TIC}

La planeación estratégica es un conjunto de decisiones y acciones que permitirán que una organización se pueda desempeñar a largo plazo, permitiendo de esta manera, que las metas propuestas por la entidad se logren ordenada y eficazmente buscando una posición competitiva en el entorno en el que desarrollan sus labores.

Esta tarea es indispensable, ya que permite identificar la razón de ser de la entidad, es decir, unifica conceptos que se van a manejar al interior de la misma, permitiendo reconocer características tan importantes como [35]:

- ¿Quiénes somos?

- ¿Qué capacidad tenemos y qué podemos hacer?

- ¿Qué problemas estamos tratando?

- ¿Qué influencia queremos causar?

- ¿Qué asuntos críticos tenemos que responder?

- ¿Dónde debemos situar nuestros recursos y cuáles son nuestras prioridades?

Después de esto, que es evaluado luego de realizar el análisis Dofa o Fado, se pueden definir la misión, la visión, los objetivos y las estrategias que permitirán realizar un seguimiento al proceso desarrollado y observar si se están obteniendo los resultados que se esperan.

\section{A. Definición de la misión y visión del Observatorio tic}

Dentro de la propuesta analizada, se realiza la construcción de la misión y la visión del observatorio así:

\section{Misión}

La misión estratégica «describe lo que hace la organización, con quién o para quién; y, grosso modo, cómo lo hace» [35], presenta la razón de ser de la organización lo que se busca conseguir, cómo funciona y en ocasiones el grupo de trabajo.

Partiendo de esos principios básicos, y sabiendo que la declaración de la misión debe indicar las razones por las cuales se constituye el Observatorio TIC de la Universidad de San Buenaventura, Bogotá, buscando mostrar de manera indirecta el objetivo principal que se pretende alcanzar, se presenta a continuación la misma:

Misión: facilitar los procesos de vigilancia, adquisición y transferencia de conocimiento y tecnología de información y comunicaciones para mejorar la competitividad de las mipymes de la localidad de Usaquén. 


\section{Visión}

La visión contiene información de "hacia dónde» quiere ir una organización, hace referencia a las proyecciones que tiene para el futuro y el lugar que quiere alcanzar con respecto a sus competidores y su posicionamiento dentro de los clientes a nivel nacional. [35].

La visión debe ser contextualizada en un marco de tiempo, a corto, mediano o largo plazo; permitiendo de esta manera, que los objetivos planteados para alcanzarla, sean razonables y percibidos como actividades realizables dentro de ese marco de tiempo.

Bajo esa premisa, la visión del Observatorio TIC de la Universidad de San Buenaventura, es la siguiente:

Visión: Para el año 2015 el Observatorio TIC será puente para construir una relación importante entre la Universidad y la empresa para la generación de nuevos proyectos, capaz de facilitar y promover el acceso al conocimiento tecnológico a través del desarrollo de soluciones en el campo de las Tecnologías de la Información y las Comunicaciones (TIC).

\section{B. Campos de trabajo del Observatorio}

El Observatorio TIC de la Universidad de San Buenaventura, está planteado para generar una amplia oferta en la que se trabajarán consultorías y oferta académica, entre la que se incluyen cursos, talleres, diplomados, foros, seminarios con las siguientes temáticas generales

- Soluciones y comunicaciones (Internet, redes alámbricas e inalámbricas, Voz IP)

- Seguridad

- Soluciones de software (Aplicaciones a la medida)

- Soluciones en hardware

- Mantenimiento de hardware.

- Gestión de la información

- Redes sociales

- Apropiación de tecnologías, entre otros

Cada una de las capacitaciones serán realizadas a la medida de las necesidades que tiene cada empresa, generando un enfoque característico que cubra las necesidades planteadas por la organización con la cual se trabaja.

A continuación se presenta una breve descripción de algunas de las temáticas que se plantea tratar dentro del Observatorio TIC: 


\section{Soluciones y comunicaciones (Internet, redes alámbricas e inalámbricas, Voz IP)}

Cada empresa tiene problemas diferentes, posee características que la hacen única, es por esta razón que necesita recursos que estén hechos a la medida, buscando satisfacer las necesidades que poseen generando soluciones integrales

La idea principal, es generar los resultados empleando diversas tecnologías, de acuerdo a los requisitos presentados por el cliente, ofreciendo soluciones en:

- Redes inalámbricas

- Redes cableadas

- Transmisión de voz

- Transmisión de datos y vídeo, entre otros

\section{Gestión de la información}

Controlar el ciclo de vida de la información que maneja la organización garantizando la integridad, disponibilidad y confidencialidad de la misma, haciendo que los procesos dentro de la organización sean más ordenados y mejorando el desempeño de los distintos departamentos que integran la entidad, facilitando la toma de decisiones en la empresa.

\section{Conjunto de posibles asociados}

De acuerdo con el análisis realizado en el capítulo 2 del presente documento acerca de los observatorios TIC existentes, se propone buscar posibilidades de convenios con los siguientes observatorios, según sus características generales:

\section{OBATIC}

Es el Observatorio Andino para las TIC tiene alcance regional en la Comunidad Andina (Colombia, Perú, Ecuador y Venezuela).

\section{Colombia Digital}

Observatorio con cobertura nacional, está impulsado por el Ministerio de las Tecnologías de la Información y las Telecomunicaciones (MinTIC)

\section{Observatorio de la Universidad Nacional}

Observatorio académico con cobertura local, impulsado por una de las universidades más importantes del país, apoyado por el grupo de Investigación Griego. Este observatorio se encuentra en etapa inicial de creación y desarrollo.

\section{Recursos claves para la creación y funcionamiento del observatorio tic}

De acuerdo con la definición dada por Farné (2010) [36] un observatorio «puede operar en virtud de simples acuerdos de voluntades entre instituciones fundadoras (participantes 
en él) o de la decisión autónoma e informal de la entidad que se hace cargo de su funcionamiento. Naturalmente, la presencia de un marco institucional que establezca fuentes de financiación, organigrama, obligaciones, objetivos y funciones de un observatorio es un claro indicador del interés por parte de las entidades patrocinadoras en formalizar, desarrollar y sostener un observatorio del mercado de trabajo».

De igual manera, un observatorio tiene como esencia cumplir con un proceso de realimentación dentro de su estructura, el cual le permita cumplir con el círculo virtuoso expuesto por Farné (2010) quien expone la necesidad de contar con un sistema de información que recopile los datos necesarios del área a trabajar, se analicen y se proponga y monitoreen propuestas a necesidades específicas divulgándolas a la comunidad:

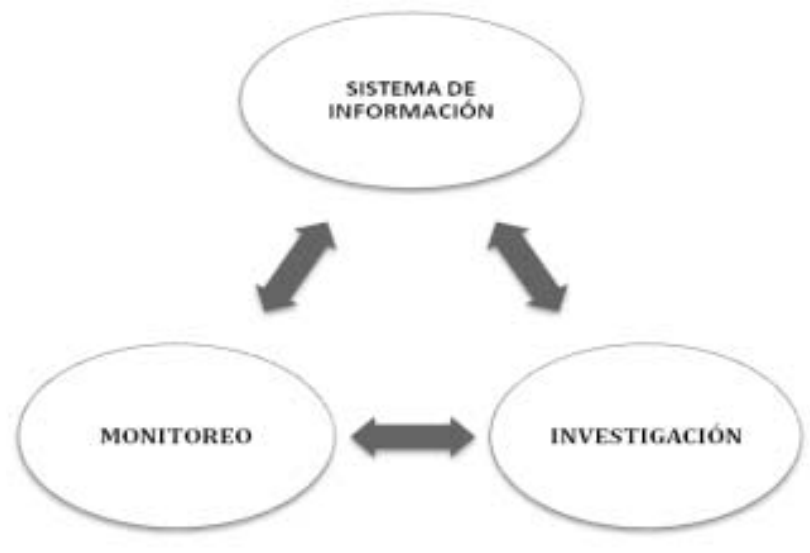

Figura 1. Círculo virtuoso [36].

De esta forma, el Observatorio TIC de la Universidad de San Buenaventura, Bogotá, será creado de acuerdo con las siguientes características:

\section{E. Estructura funcional y operativa del Observatorio}

Un observatorio es un instrumento de apoyo y de complementación al ejercicio investigativo que realiza la Universidad con respecto a la apropiación de las TIC a nivel de las mypimes. Se proyecta como un sistema de recolección y análisis de información que permite realimentar y proponer soluciones tecnológicas para las empresas analizadas y posibles soluciones para otras mypimes. Para ello es necesario contar con una estructura organizacional que permita alcanzar los objetivos y metas planteados, por ser un proyecto en construcción, el éxito depende del adecuado dimensionamiento de la estructura funcional.

Aunque inicialmente la única figura permanente del observatorio serán los integrantes que la institución delegue, se cuenta de igual manera con una variedad multidisciplinar de profesionales quienes desde su saber particular aportarán y alimentarán el observatorio impulsando los proyectos propuestos; este equipo será el encargado de realizar investigaciones básicas de evaluación de apropiación y uso de las TIC en las mipymes, realizar las propuestas de implementación de acuerdo con dichos estudios y coordinar y ejecutar las diferentes labores administrativas propias del observatorio (elaboración de cartas, redacción de documentos, etc.). Se propone entonces la siguiente estructura funcional: 


\section{Coordinador del observatorio}

Es la cabeza del observatorio y estará encargado de planificar, dirigir, coordinar y supervisar informes, análisis e investigaciones del mercado de mipymes y de los aspectos que inciden en sus características teniendo en cuenta la información de apropiación y uso de las TIC.

\section{Comité de investigación e información}

Teniendo en cuenta que la disponibilidad de información es fundamental para la planificación y diseño de estrategias y acciones de implementación de herramientas TIC en las empresas, es necesario contar con una división encargada de la búsqueda y consolidación de la información correspondiente a cada una de las áreas de trabajo del observatorio la cual debe ser confiable, adecuada y actualizada para permitir la toma de decisiones y la correcta elaboración de los planes y proyectos propuestos. Dentro de sus funciones se encuentran entre otras las siguientes: realizar estudios e investigaciones sobre las mipymes y su uso y apropiación de las TIC aplicando estadísticas e indicadores, consultar y manejar las herramientas TIC actuales y futuras aplicables a mipymes manteniendo actualizado el observatorio usando fuentes tanto internas como externas.

\section{Comité de análisis y desarrollo}

La información recolectada debe ser almacenada, clasificada y analizada adecuadamente con el fin de construir las propuestas necesarias según el perfil de la empresa estudiada e incorporando a los aliados que tengan injerencia en el área trabajada. Tiene entre sus principales funciones realizar análisis sobre las mipymes en cuanto uso y apropiación de las TIC.

\section{Comité de análisis y producción de documentos}

La comunicación de los resultados es clave para fortalecer los procesos y repotenciar los resultados, este comité es el encargado de realizar el análisis y la producción de documentos correspondientes a la divulgación de las actividades propias del Observatorio. Para tal fin, se propone la creación de una página web, la cual sirva como sitio de información específica y actualizada acerca de las investigaciones, desarrollos y avances de los proyectos relacionados con las áreas de trabajo del observatorio.

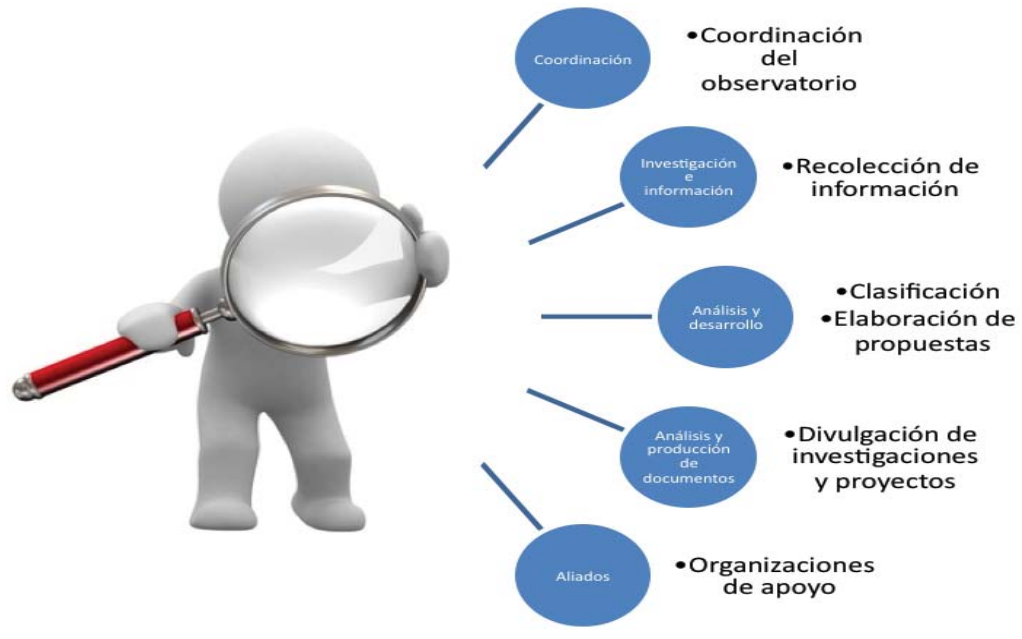

Figura 2. Estructura Observatorio TIC. Fuente: elaboración propia. 


\section{F. Vinculación del Observatorio tic al sistema nacional de ciencia, tecnología e innovación}

Para vincular el observatorio se tienen dos opciones, que no son obligatorias dado que Colciencias, en su página web aduce la no necesidad de registro del Observatorio, y, que únicamente es necesario adelantar proyectos que trabajen temáticas de ciencia y tecnología [37].

Las dos vías de acción, para la vinculación del observatorio al SNCTI, son:

- Registrar el observatorio como independiente

- Registrarlo como grupo de investigación

Para las dos opciones se presentan los requisitos básicos necesarios para el registro ante Colciencias.

\section{Observatorio TIC como independiente}

En este caso, se hace referencia a que va a ser una entidad totalmente independiente de la Universidad de San Buenaventura, para lo cual es necesario:

- Crear y formalizar la figura jurídica de la entidad, esto se realiza ante la Cámara de Comercio de Bogotá.

- Registrar el Observatorio TIC en la plataforma de Colciencias, a través del formulario digital para Reconocimiento de Centros, que se encuentra en la página web: www. colciencias.gov.co/formularios_sigp; en ella se encuentra el vínculo de descarga de la herramienta que permite hacer el registro ante Colciencias.

- Anexar documentación exigida por Colciencias, de acuerdo a la Resolución 688 de 2012, que establece definiciones y requisitos para el reconocimiento de centros de investigación o desarrollo tecnológico [38].

\section{Observatorio tic como Grupo de Investigación}

Cuando se hace este registro, se hace la vinculación del Observatorio a la plataforma GrupLAC, asociándolo a la Universidad de San Buenaventura.

En cualesquiera de los dos casos, es necesario que los integrantes que sean líderes del Observatorio, tengan sus hojas de vida registradas ante CVLAC, ya que se requiere que los integrantes del Observatorio, demuestren que son personal calificado a nivel de investigación para desarrollar las diferentes actividades que se realicen al interior del mismo, garantizando de esta manera, un mayor reconocimiento a las labores realizadas.

En cualesquiera de las dos modalidades, el Observatorio TIC, podrá presentarse a las convocatorias que periódicamente publica Colciencias, siempre y cuando, cumpla con los requisitos básicos exigidos dentro del marco de referencia de la convocatoria en cuestión. 


\section{G. Metodologías de trabajo del observatorio tic}

Teniendo en cuenta que el Observatorio TIC, es una organización que propende el trabajo con las mipymes del sector de Toberín, es necesario identificar los elementos y factores que generan mayor valor al interior de las organizaciones $y$, se espera, mayor competitividad; por esta razón es importante realizar un seguimiento y acompañamiento a las mismas a partir de modelos o metodologías que faciliten esta actividad por parte del Observatorio TIC.

Los procesos que se pretende realizar al interior del Observatorio TIC son:

- Vigilancia tecnológica

- Transferencia tecnológica

- Prospectiva tecnológica

\section{Vigilancia tecnológica}

Al hablar de vigilancia tecnológica, se busca conocer las tecnologías actuales y emergentes, manteniendo a la vanguardia las tecnologías que hay al interior de la organización, buscando o detectando cambios de tendencias y nuevas tecnologías para prepararse ante ellos; además permite identificar amenazas y oportunidades que aporten a las estrategias que minimicen los riesgos de la empresa.

Existen tres maneras de realizar vigilancia tecnológica [39]:

- Vigilancia pasiva (Scanning): descubrir información de interés para la empresa.

- Vigilancia activa (Monitoring): buscar información de manera regular, brindando conocimiento actualizado. En ocasiones se puede centrar en un tema determinado.

- Watching: incluye las vigilancias pasiva y activa. Incorpora observación, análisis y difusión.

Teniendo en cuenta lo anterior, su busca con la vigilancia tecnológica que la empresa tenga la capacidad de tomar decisiones estratégicas.

\section{Apropiación tecnológica}

En este caso, se hace referencia al proceso que hace cada persona para apropiarse de una tecnología, a partir de uso e incorporación a las actividades diarias. Esto implica también que se fortalezcan las habilidades del personal a nivel tecnológico, racional y cognitivo [40].

Cabe aclarar que el proceso que se desarrolla es totalmente dependiente del individuo y de qué tanto interés tenga este por acercarse a la tecnología; hay que tener en cuenta que este es un proceso netamente subjetivo y será realmente visible si el individuo hace uso de la tecnología con mayor frecuencia, teniendo en cuenta lo anterior, no se presenta o genera un modelo que muestre las diferentes fases de la apropiación tecnológica. 


\section{Prospectiva tecnológica}

Elabora el futuro, partiendo de una serie de opciones, todas válidas, de sucesos que han sido diseñados y evaluado como posibles [41].

Al evaluar una serie de opciones, la prospectiva tecnológica no brinda una sola solución, es decir, presenta diferentes soluciones, que después de analizar detenidamente, puede llevar a una única solución; esto con el fin de que la organización esté en capacidad de prepararse ante distintos escenarios que puedan llegar a presentarse y poder escoger el mejor, facilitando la toma de decisiones.

\section{Conclusiones}

Al ser las TIC un elemento transversal se hace necesario concientizar e involucrar a todos los agentes sociales y empresariales en su relevancia e importancia en la competitividad empresarial y el desarrollo social del país mediante programas de acompañamiento tecnológico y creación y/o modificación de políticas nacionales e internacionales.

Los diferentes procesos de innovación permiten generar nuevas y variadas herramientas TIC las cuales posibilitan que cualquier empresa pueda incorporarlas en mayor o menor medida en todos sus procesos y de acuerdo con sus capacidades y necesidades; la clave está en elegir la herramienta adecuada para cada necesidad.

La incorporación de las TIC debe hacerse de una manera progresiva y ordenada acompañada de un plan de capacitación y un cambio cultural que permitan una adecuada asimilación de los nuevos procesos y uso de las nuevas herramientas seleccionadas.

Debido a las diferentes naturalezas de las organizaciones, el plan de incorporación de TIC debe realizarse mediante una identificación de necesidades específicas y una concientización y sensibilización proponiendo soluciones a la medida de las empresas realizando un acompañamiento personalizado a dicho proceso.

Los procesos de vigilancia, transferencia y prospectiva tecnológica son procesos emergentes que permitirán fortalecer los procedimientos que desarrollados al interior de las empresas, mejorando de esta manera la toma de decisiones y permitiendo que las mipymes se conviertan en entidades más competitivas.

\section{Referencias}

[1] Ley 590 de 2000. Por la cual se dictan disposiciones para promover el desarrollo de las micro, pequeñas y medianas empresas. Congreso de la República de Colombia.

[2] Tomado y adaptado de la Ley 905 de 2004. Por medio de la cual se modifica la Ley 590 de 2000 sobre la pro promoción del desarrollo de la micro, pequeña y mediana empresa colombiana y se dictan otras disposiciones. Congreso de la República de Colombia.

[3] Ley 905 de 2004. Por medio de la cual se modifica la Ley 590 de 2000 sobre promoción del desarrollo de la micro, pequeña y mediana empresa colombiana y se dictan otras disposiciones. Congreso de la República de Colombia. 
[4] ACM (Associationfor Computing Machinery), Asociación de profesionales e investigadores en temas relacionados con las ciencias de la computación, que propone estándares de reconocimiento mundial.

[5] Traducción del original: The IEEE Standard Dictionary of Electrical and Electronic Terms. $6^{\text {th }}$ Edición. New York: Editorial IEEE Standards Office, 1997. ISBN: 1559378336.

[6] Estado actual de la Ingeniería de Sistemas en el país [En línea]. Bogotá: Redis, 2011 [Consultado 2013-04-16]. Disponible en Internet: http://rediscol.org/cmsv3/?p=223\#more-223

[7] Recomendación UIT-R V.662-3 (2000) [Consultado 2013-04-16]. Disponible en Internet: www.itu.int/dms_pubrec/itu-r/ $\mathrm{rec} / \mathrm{v} / \mathrm{R}-\mathrm{REC}-\mathrm{V} .662-3-200005-$ I!!pdf-s.pdf

[8] Dane, CIUO-88 A.C.

[9] Chapman, A. (2004). Análisis DOFA y análisis Pest. [Consultado 2013-04-16]. Disponible en Internet: http://lulsam.no-ip. org/backup/esucs-sampedm/datos/info/DAFO-PEST/An\%C3\%A1lisis\%20DOFA\%20y\%20an\%C3\%A1lisis\%20pest.doc

[10] Thompson Jr. A. Administración estratégica, teoría y casos.

[11] Observatorio Nacional de Tecnologías de la Sociedad de la Información, Ministerio de Industria, Energía y Turismo. España. [Consultado: 201303 27]. Disponible en www.ontsi.red.es/ontsi/

[12] Cesalvi El observatorio Valenciano para la Sociedad Tecnológica y del Conocimiento [Consultado 2013032 Disponible en <ftp://ftp.educa.madrid.org/pub/Miscelanea/IXJornadasTI/03T_PDF/cevalsi\%20el\%20observatorio\%20valenciano.pdf >I

[13] Observatorio Aragonés de la Sociedad de la Información, Departamento de Industria e Innovación, Gobierno de Aragón. [Consultado 2013-03- 27] Disponible en www.observatorioaragones.org/

[14] Instituto Nacional de las Tecnologías de Comunicación. Gobierno de España. [Consultado: 2013-03-29]. Disponible en www. inteco.es/

[15] Ministerio de Ciencia, Tecnología e Innovación Productiva. [Consultado 2013-03-29]. Disponible en www.mincyt.gov.ar/ noticias/index.php

[16] Observatorio de la Sociedad de la Información en Navarra, Programa de acciones innovadoras de Navarra 2000. [Consultado: 2013-03-29]. Disponible en <www.cfnavarra.es/observatoriosi/praina.htm>

[17] Observatorios de la Unesco. [Consultado 2013-04-02]. Disponible en www.portal.unesco.org/ci/en/ev.phpURL_ID=7277\&URL_ DO=DO_TOPIC\&URL_SECTION=201.html

[18] Observatorio de las Tecnologías de la Información y de la Comunicación (OTIC). [Consultado 2013-04-02]. Disponible en <https://sites.google.com/site/oticarmilla/>

[19] El Observatorio de la Sociedad de la Información y el Conocimiento de República Dominicana (OSIC-RD). [Consultado 201304-02]. Disponible en http://osicrd.one.gob.do/

[20] Observatorio Andino de las mipymes. [Consultado: 2012-04-02]. <http://observatorioredesempresariales.wordpress. com/2012/07/05/paises-de-la-can-lanzan-observatorio-andino-de-la-mipyme/>

[21] Colombia Digital, Somos la Corporación Colombiana: Colombia Digital. [Consultado: 2013-04-02]. Disponible en www. colombiadigital.net/corporacion-colombia-digital/somos-la-corporacion-colombia-digital.html

[22] Universidad Nacional creará Observatorio Nacional de Tecnologías en pymes. [Consultado: 2013-04-27]. Disponible en <http://noticias.universia.net.co/en-portada/noticia/2011/01/12/778895/universidad-nacional-creara-observatorionacional-tecnologias-pymes.html>

[23] Observatorio del Caribe Colombiano. [Consultado: 2013-04-27]. Disponible en www.ocaribe.org/

[24] Medellín Digital, El Observatorio. [Consultado: 2013-04-02]. Disponible <www.medellindigital.gov.co/nuestraestrategia/ MedellinCiudaddelConocimiento/Paginas/Observatorio.aspx>

[25] Observatorio de Pedagogía y Educación. [Consultado: 2013-04-27]. Disponible en www.idep.edu.co/cartografia/opes/index.html

[26] Observatorio de Sociedad, Gobierno y Tecnologías de la Información (TIC): OOBSERVATIC [Consultado: 2013- 04-02]. Disponible en <http://observatics.edu.co/vision-y-mision-observatics/>

[27] Presentación del Observatorio Colombiano de Ciencia y Tecnología (OCyT) [Consultado 2013-04-02]. Disponible en http:// ocyt.org.co/html/index.php?option=com_content\&view=article\&id=45\&ltemid=58\&lang=es

[28] Las economías latinoamericanas todavía están atrasadas en el aprovechamiento de las tecnologías de la información y las comunicaciones, a pesar de estar avanzando según un nuevo informe global sobre tecnología. [Consultado 2013-04-26] Disponible en: www.weforum.org/news/las-econom\%C3\%ADas-latinoamericanas-todav\%C3\%ADa-est\%C3\%A1 n-atrasadasen-el-aprovechamiento-de-las-tecnolog\%C3\%ADas

[29] El Banco Mundial. [Consultado: 2013-04-26]. Disponible en:<http://web.worldbank.org>

[30] Unión internacional de telecomunicaciones. [Consultado: 2013-04-26]. Disponible en: www.itu.int/en/about/Pages/overview.aspx

[31] Plan Nacional de Tecnologías de la Información y las Comunicaciones, Ministerio de Comunicaciones 2008-2019. República de Colombia, Bogotá marzo 2008. [Consultado 2013-04-08]. Disponible en Internet: www.medellin.edu.co/sites/Educativo/ Directivos/Noticias/Paginas/ED11_RD_PlanNacionaldeTIC.aspx 
[32] Plan Vive Digital. [Consultado 2013-04-26]. Disponible en: <www.mintic.gov.co/images/MS_VIVE_DIGITAL/archivos/ Vivo_Vive_Digital.pdf>

[33] Política Nacional de Productividad y Competitividad. [Consultado: 2013-04-24]. Disponible en: www.mincomercio.gov.co/ publicaciones. php?id=14894

[34] Sistema Nacional de Ciencia, Tecnología e Innovación, Ley 1286 de 2009.

[35] Elabora la planeación estratégica para tu empresa [en línea]. [Consultado: 2013 -05-13]. Disponible en: www.bogotaemprende. com/documentos/5026_Doc_resumen_Planeaci\%C3\%B3n_estrat\%C3\%A9gica.pdf

[36] Farné, S. Observatorios del mercado de trabajo: ¿Qué son y cómo funcionan? [Consultado: 2013 -07-26]. Disponible en:http://190.7.110.123/pdf/6_derechoSeguridadSocial/observatorio/Que_es_observatorio.pdf

[37] Colciencias. Preguntas frecuentes. Disponible en:<www.colciencias.gov.co/faq >. Consultado el: 26 de agosto de 2013.

[38] Colciencias. Resolución 688 de 2012 por la cual se deroga Resolución 504 de 2012 y se establecen definiciones y requisitos para el reconocimiento de los centros de investigación o desarrollo tecnológico. Disponible en:<www.colciencias.gov.co/ sites/default/files/ckeditor files/files/Resoluci\%C3\%B3n\%20688-2012.pdf>. Consultado el: 26 de agosto de 2013.

[39] Castellanos Domínguez, Óscar Fernando. Gestión tecnológica. De un enfoque tradicional a la inteligencia. Universidad Nacional de Colombia, p. 189.

[40] Ochoa Urrego, Rafael Leonardo. ¿Asimilación, adopción o apropiación? Un acercamiento conceptual al fenómeno de la Inclusión Tecnológica.

[41] Castellanos Domínguez, Óscar Fernando. Gestión tecnológica. De un enfoque tradicional a la inteligencia. Universidad Nacional de Colombia, p.199.

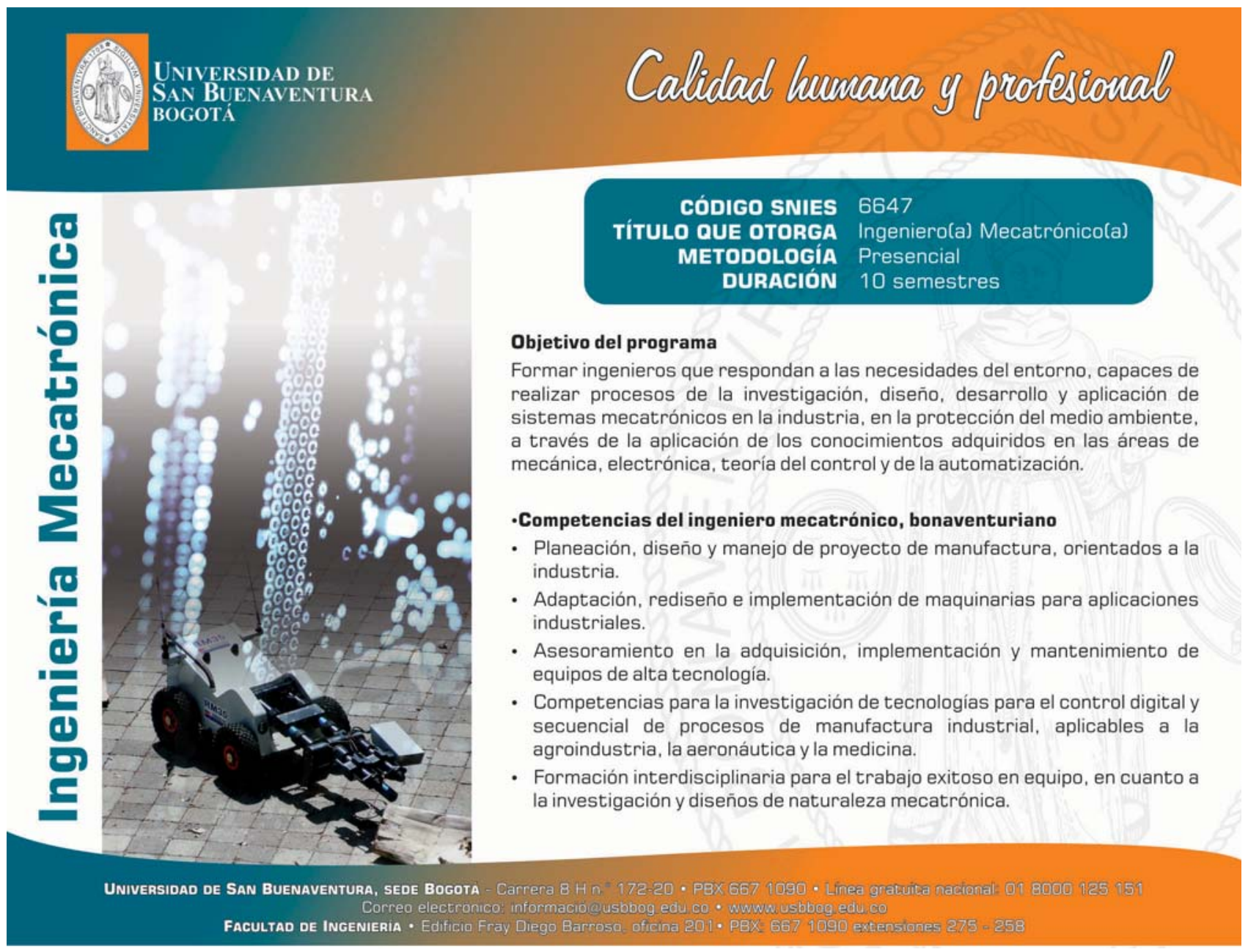

